\title{
A Tunable Polarization-Dependent Terahertz Metamaterial Absorber Based on Liquid Crystal
}

\author{
Guangsheng Deng ${ }^{1}{ }^{(0)}$, Yujiao Lu ${ }^{1}$, Zhiping Yin ${ }^{1}$, Weien Lai ${ }^{1}$, Hongbo Lu ${ }^{1}{ }^{(}$, Jun Yang ${ }^{1, *(1)}$, \\ Aifeng Yang ${ }^{2}$, Yang $\mathrm{Ye}^{3}$, Dayong $\mathrm{Liu}^{3}$ and Baihong $\mathrm{Chi}^{3}$ \\ 1 National Key Laboratory of Advanced Display Technology, Academy of Photoelectric Technology, \\ Hefei University of Technology, Hefei 230009, China; dgsh@hfut.edu.cn (G.D.); yjlu@mail.hfut.edu.cn (Y.L.); \\ zpyin@hfut.edu.cn (Z.Y.); bluecpu05@163.com (W.L.); bozhilu@hfut.edu.cn (H.L.) \\ 2 School of Management, Hefei University of Technology, Hefei 230009, China; aifengy@163.com \\ 3 Process and Mechanical Engineering Technology Laboratory, Space Star Technology Co. Ltd., Beijing 100095, \\ China; yangtze_2008@sina.com (Y.Y.); liudywork@sina.com (D.L.); baihong1988@163.com (B.C.) \\ * Correspondence: junyang@hfut.edu.cn
}

Received: 19 January 2018; Accepted: 23 February 2018; Published: 26 February 2018

\begin{abstract}
In this paper, a tunable polarization-dependent terahertz (THz) metamaterial absorber based on liquid crystal (LC) is presented. The measurement results show that absorption peak is at $239.5 \mathrm{GHz}$ for a TE-polarized wave and $306.6 \mathrm{GHz}$ for a TM-polarized wave, without exerting the bias voltage on the LC layer. An increase in bias voltage affects the orientation of LC molecules and causes redshifted resonant frequencies. By adjusting the bias voltage from 0 to $10 \mathrm{~V}$, frequency tunabilities of $4.7 \%$ and $4.1 \%$ for TE- and TM-polarized waves, respectively, were experimentally demonstrated. Surface current and power loss distribution was analyzed to explain the physical mechanism of the absorber, while the absorption dependence on geometrical parameters and incident angles was also studied in detail. According to the obtained results, the proposed absorber is shown here to be capable of achieving tunable polarization-dependent absorption, and to have potential application in terahertz polarization imaging, terahertz sensing, and polarization multiplexing.
\end{abstract}

Keywords: metamaterials; liquid-crystal devices; polarization-selective devices

\section{Introduction}

In the last decade, terahertz (THz) technology, which has great potential in sensing, imaging, and spectroscopy, has attracted attention [1,2]. Among the emerging THz devices, THz absorbers play an important role in several THz systems, including sensors, stealth materials, and detectors [3-5].

Since Landy et al. presented the concept of a metamaterial absorber (MA) in 2008 [6], MA has attracted great interest due to its enormous advantages such as low cost, small size, and ultrathin thickness. Various MAs, such as wideband absorbers [7-9] and multiband absorbers [10-12], have been widely investigated in recent years. In many applications, the polarization-dependent absorption is useful and demanded. The polarization information can further increase the depth accuracy of detected images [13] and is of great significance in remote sensing, biomedical imaging, and image sensors. Sakurai et al. proposed an L-shaped polarization-dependent MA in the infrared region [14]. Wang et al. presented a polarization tunable terahertz MA based on an asymmetric metallic resonant patch [15]. Kim et al. realized a dual-absorption polarization sensitive metamaterial structure by composing four cut-wire bars [16].

However, to the best of the authors' knowledge, for a given polarization angle, most of the existing polarization-dependent absorbers show characteristics of perfect absorption only at certain frequency. Although absorbers can be effectively tuned to different working frequencies by reconstructing the geometries, it is very difficult to change the physical structure after fabrication. In addition, 
for some sensing and detecting applications, a tunable absorber, whose operation frequency can be tuned within a certain frequency range, is essential and necessary. Ho et al. introduced electrothermally actuated microactuators integrated with an Omega-ring metamaterial unit cell to provide continuous tuning [17], but the fabrication of this structure was complicated. In our previous work, a polarization-dependent tunable terahertz MA based on a graphene monolayer was presented [18]. Nevertheless, acquiring a perfect monolayer graphene sheet is still a challenge.

It has been verified that the absorption frequency of an MA based on liquid crystal (LC) can be dynamically tuned in the terahertz region due to the voltage-dependent birefringence of LC $[19,20]$. Based on this concept, a polarization-dependent LC tunable MA at terahertz frequencies based on a new metamaterial unit cell is proposed in this paper. The influence of the orientation on LC cells on the absorber's absorption spectrum in two orthogonal directions is theoretically investigated. It is shown that a distinct absorption peak with no bias voltage is located at $239.5 \mathrm{GHz}$ for TE-polarization and $306.6 \mathrm{GHz}$ for TM-polarization. Moreover, it is experimentally demonstrated that, by controlling the bias voltage of LC molecules, the absorption peak can be tuned with a maximal frequency tunability of $4.7 \%$ and $4.1 \%$ for TE- and TM-polarization, respectively.

\section{Structure Design and Principle of Operation}

The structure of the unit cell of the proposed polarization-dependent tunable absorber is presented in Figure 1. This unit cell is composed of a pair of metal layers printed on the surface of parallel quartz plates with a void between them, which is fully filled with a nematic liquid crystal. The metallic patterned layer at the upper surface of the quartz plate serves not only as a resonant structure, which shows characteristics of polarization dependence, but also as a top electrode of the whole tunable absorber.
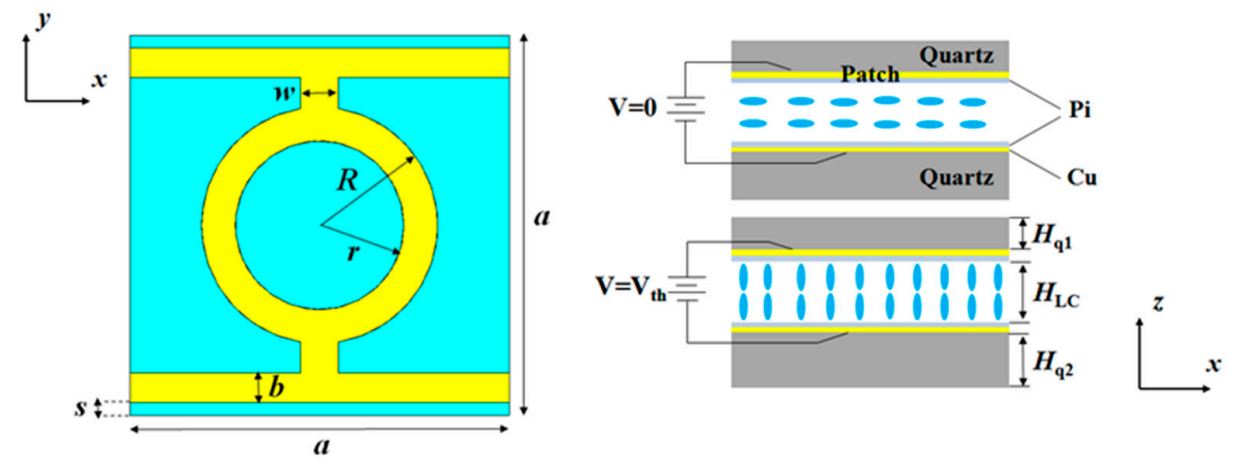

Figure 1. Schematic diagram of a metamaterial absorber based on liquid crystal (LC).

It is well known that LC materials are composed of anisotropic molecules and that the orientation of LC molecules can be controlled with an external electrical field. Thus, by changing the bias voltage between two metal layers, the direction of LC molecules along the electric field vector of the incident wave, i.e., the permittivity of the LC layer, can be dynamically adjusted. To control the direction of LC molecules without applying a bias voltage, a thin layer of polyimide ( $\mathrm{Pi}$ ) is spanned on the inner surface of both metal layers. In the unbiased state $\left(\varepsilon_{\perp}\right)$, due to the static action of the polymer alignment layer, the long axis of molecules is parallel to the quartz surface. The bias voltage, which is applied across the biasing layers, rotates the LC molecules, which provides the maximal permittivity $\left(\varepsilon_{\mid l}\right)$ at the saturation state, while the direction of the LC molecules is perpendicular to the cell walls. Therefore, the LC permittivity can be electronically varied between these two states, i.e., $\Delta \varepsilon_{\text {eff }}=\varepsilon_{||}-\varepsilon_{\perp}$, thus the absorption frequency of the absorber can be shifted.

The geometrical dimensions of the unit-cell structure presented in Figure 1 are as follows: $\mathrm{a}=460 \mu \mathrm{m}, \mathrm{b}=35 \mu \mathrm{m}, \mathrm{R}=135 \mu \mathrm{m}, \mathrm{r}=100 \mu \mathrm{m}, \mathrm{w}=45 \mu \mathrm{m}, \mathrm{s}=15 \mu \mathrm{m}, \mathrm{H}_{\mathrm{q} 1}=350 \mu \mathrm{m}, \mathrm{H}_{\mathrm{q} 2}=1000 \mu \mathrm{m}$, and $\mathrm{H}_{\mathrm{LC}}=45 \mu \mathrm{m}$. Both metal layers are made of a $0.5-\mu \mathrm{m}$-thick copper layer with a conductivity of 
$\sigma=5.8 \times 10^{7} \mathrm{~S} / \mathrm{m}$, and quartz plates are considered as a dielectric material with a relative permittivity of 3.78 and a dielectric loss tangent of 0.02 . The absorptivity of an absorber can be determined by $A=1-\left|S_{11}\right|^{2}-\left|S_{21}\right|^{2}$, where $\mathrm{A}, \mathrm{S}_{11}$, and $\mathrm{S}_{21}$ denote the absorptivity, reflection coefficient, and transmission coefficient, respectively. Since the transmission is equal to zero due to the existence of the copper ground plate, the absorptivity can be simplified as $A=1-\left|S_{11}\right|^{2}$.

\section{Simulated Results and Absorption Mechanisms}

The simulations were conducted by using the finite-element frequency-domain method, and the unit-cell boundary conditions were utilized in both the $x$ and $y$ directions. Meanwhile, the Floquet port condition was employed in the $z$-direction. According to our previously reported results [21], the LC material was assumed to be S200 in the simulations, where its permittivity in the unbiased state $\left(\varepsilon_{\perp}\right)$ and the fully biased state $\left(\varepsilon_{\mid}\right)$was set to 2.47 and 3.06 , respectively. In the simulation, the loss tangent of the LC layer is set to 0.02 . Moreover, in this study, the LC is treated as a homogeneous material, which is widely used in the modeling of LC-based devices [22-24], and the effective index of the aligned nematic LC is given by [25]

$$
\mathrm{n}_{\mathrm{eff}}=\frac{\mathrm{n}_{\mathrm{e}} \mathrm{n}_{\mathrm{o}}}{\sqrt{\mathrm{n}_{\mathrm{e}}^{2} \sin ^{2} \theta+\mathrm{n}_{\mathrm{o}}^{2} \cos ^{2} \theta}}
$$

where $\mathrm{n}_{\mathrm{o}}$ and $\mathrm{n}_{\mathrm{e}}$ represent the ordinary and extraordinary indices, respectively, and $\theta$ is the LC director angle. Since the LC is a nonmagnetic material, (1) can be rewritten by

$$
\varepsilon=\frac{\varepsilon_{\perp} \varepsilon_{/ /}}{\varepsilon_{\perp} \sin ^{2} \theta+\varepsilon_{/ /} \cos ^{2} \theta} .
$$

As shown in Figure 2, it can be noticed that, for both TE- and TM-polarized wave incidences, the direction of the electric field vector in the LC layer is mainly parallel to the $z$-axis, which is perpendicular to the direction of LC molecules. Hence, the LC permittivity is equal to $\varepsilon_{\perp}$ in the initial case $\left(\theta=0^{\circ}\right)$. With the increase in bias voltage applied on the LC layer, the LC permittivity increased to $\varepsilon_{\mid}$। in the fully biased state, and the reorientation angle of the LC molecule gradually shifted toward the $z$-axis and was finally parallel to the $z$-axis $\left(\theta=90^{\circ}\right)$.

(a)
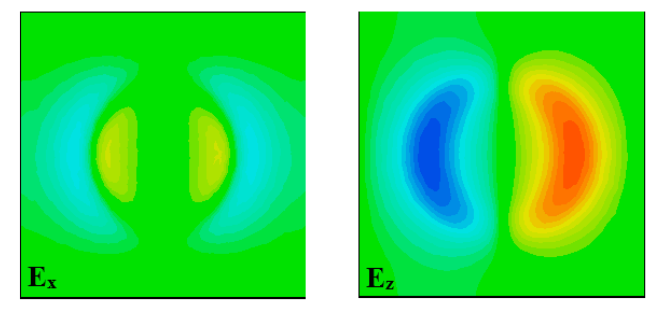

0

$7.73 e 5 \mathrm{~V} / \mathrm{m}$

(b)
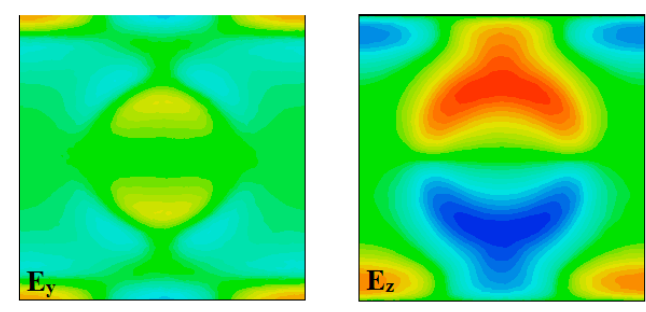

0

$5.84 \mathrm{e} 5 \mathrm{~V} / \mathrm{m}$

Figure 2. Calculated results for peak absorption frequency: (a) distribution of the electric field in the LC layer under TE-polarized normal incidence at $238.4 \mathrm{GHz}$; (b) distribution of the electric field in the LC layer under TM-polarized normal incidence at $313.2 \mathrm{GHz}$. 
The absorption spectra for TE-polarized and TM-polarized normal incidence excitations for different LC director angles, which demonstrate the tunable performance of the proposed LC-based metamaterial absorber, were calculated and they are shown in Figure 3. In Figure 3, it can be seen that, as the LC director angle increases from 0 to $90^{\circ}$, the distinct peak absorption frequency for TE-polarized incidence changes from 238.4 to $226.1 \mathrm{GHz}$, with a frequency tunability $\left(\mathrm{f}_{\bmod }=\Delta \mathrm{f} / \mathrm{f}_{\max }\right)$ of $5.1 \%$. Meanwhile, a frequency tunability of $5.7 \%$ is achieved for TM-polarized incidence as the peak absorption frequency varies from 313.2 to $295.2 \mathrm{GHz}$. Moreover, it can also be seen in Figure 3 that, for both TE-polarized and TM-polarized incidence excitations, absorptivity remains higher than $90 \%$ (except for the LC director angle of $0^{\circ}$ for TE-polarization), so the proposed metamaterial absorber possesses high absorption within the entire tunable frequency region, a promising finding.

(a)

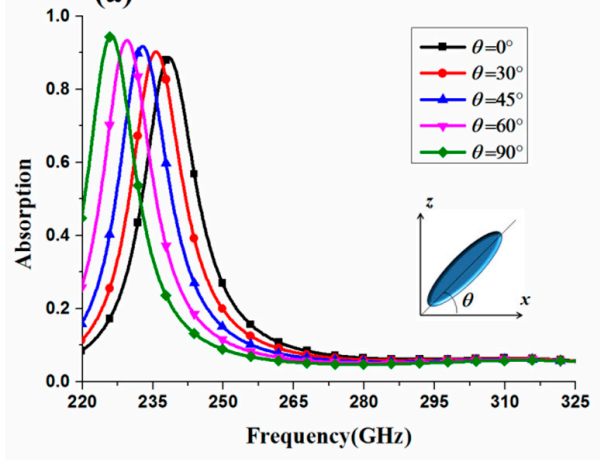

(b)

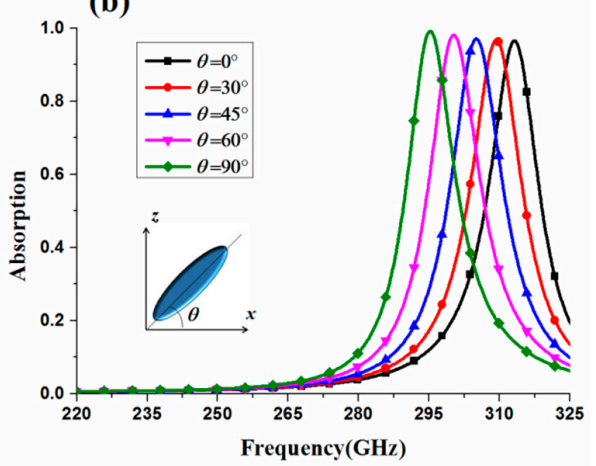

Figure 3. Simulated absorption spectra of absorber for different LC director angles for (a) the TE-polarized incident wave and (b) the TM-polarized incident wave.

To explore the intrinsic mechanism of the absorber, the surface current distribution on the patterned copper layer and the copper ground plate for the TE-polarized and TM-polarized normal incidence excitations in the unbiased state was calculated and is illustrated in Figure 4.
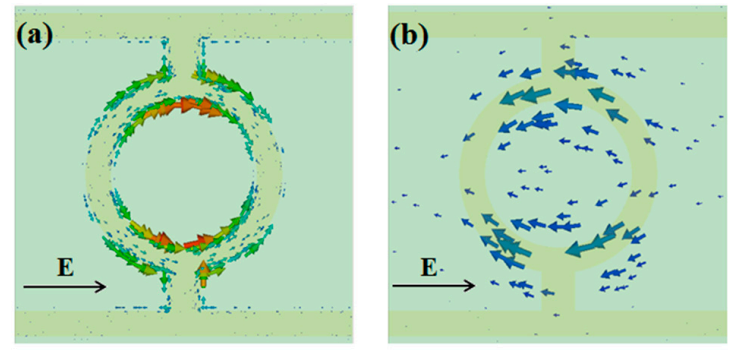

0

$21714(\mathrm{~A} / \mathrm{m})$
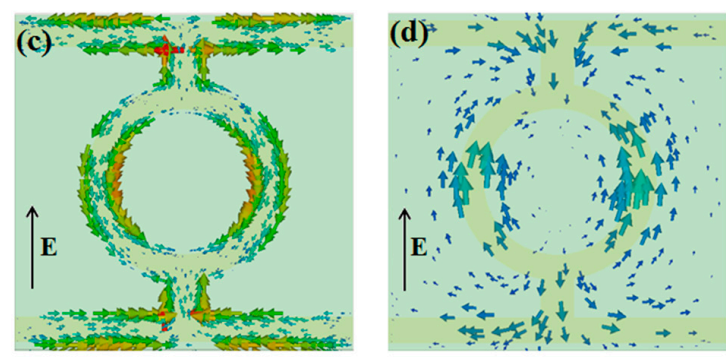

0

$22670(\mathrm{~A} / \mathrm{m})$

Figure 4. Simulated surface current distribution on (a) the patterned copper layer at $238.4 \mathrm{GHz}$ under TE-polarized incidence, (b) the copper ground plane at $238.4 \mathrm{GHz}$ under TE-polarized incidence, (c) the patterned copper layer at $313.2 \mathrm{GHz}$ under TM-polarized incidence, and (d) the copper ground plane at $313.2 \mathrm{GHz}$ under TM-polarized incidence. 
According to the results presented in Figure $4 \mathrm{a}$, it is obvious that the surface current on the copper pattern flows parallel to the direction of the electric field vector of TE-polarized incident wave. As a result, two electric dipole responses are excited in the patterned layer. Moreover, the surface current on the copper ground plate shown in Figure $4 \mathrm{~b}$ is the reverse of that in Figure $4 \mathrm{a}$, as the magnetic response is excited by circulating currents between the two metallic layers. For the TM-polarized incident wave, as shown in Figure 4c, four dipoles are excited, and the electric response is mainly contributed by coupling interaction between these dipoles. Similarly, the surface current on the copper ground plane is reversed, and the magnetic response for TM-polarized incident wave is also excited by combining the copper pattern with the copper plate.

Furthermore, the ohmic losses at the patterned copper layer, as well as the power loss density in the quartz plate and the LC layer, for both the TE- and TM-polarized incident waves were calculated and are illustrated in Figure 5. The dielectric loss distribution of the absorber is in agreement with the electric field distribution, and the ohmic loss distribution at the pattern surface is mainly determined by the surface currents. In addition, it can be observed that most of the energy in the absorber is dissipated in the LC layer, which contributes most of the power absorption under both TE- and TM-polarized normal incidences. Thus, the LC plays an important role not only in frequency tuning but also in the absorption of incident waves.

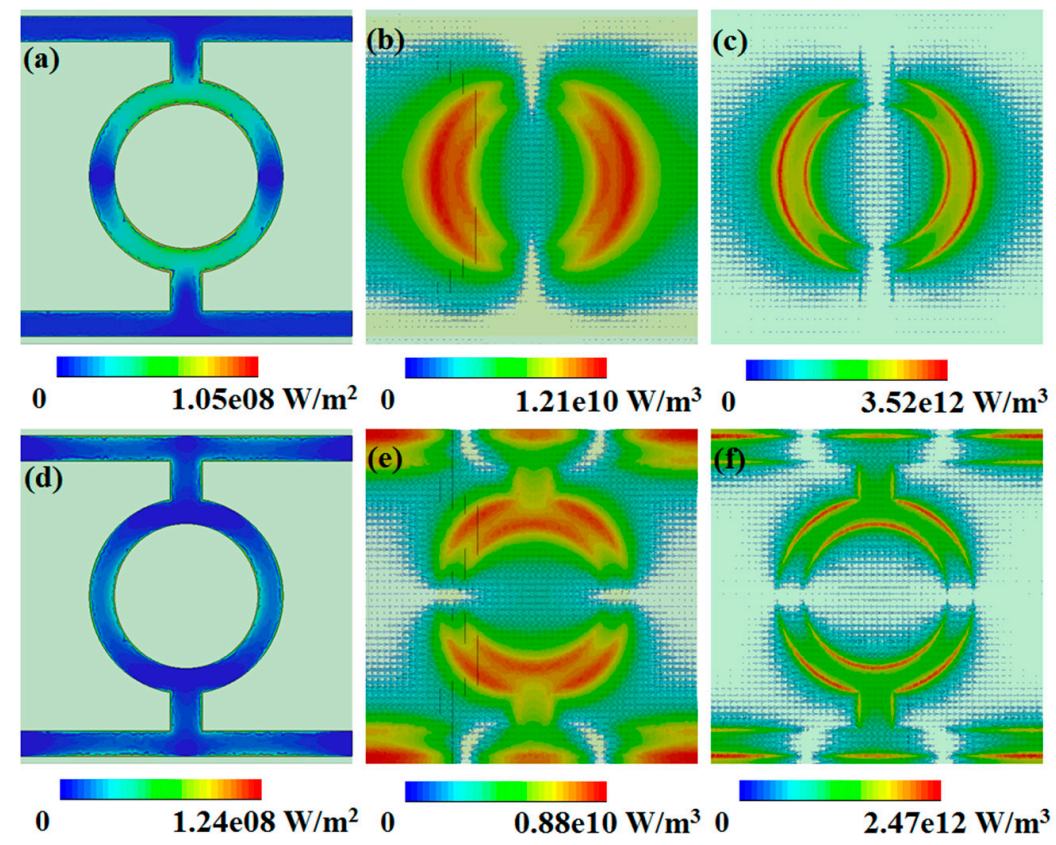

Figure 5. Calculated results for peak absorption frequency: (a) distribution of surface losses at the patterned copper layer at $238.4 \mathrm{GHz}$, (b) distribution of dielectric power loss density of the quartz plate at $238.4 \mathrm{GHz}$, (c) distribution of dielectric power loss density of the LC layer at $238.4 \mathrm{GHz}$, (d) distribution of surface losses at the patterned copper layer at $313.2 \mathrm{GHz}$, (e) distribution of dielectric power loss density of the quartz plate at $313.2 \mathrm{GHz}$, and (f) distribution of dielectric power loss density of the LC layer at $313.2 \mathrm{GHz}$.

\section{Absorption Spectrum Dependence on Geometrical Parameters and Incident Angle}

In this section, the effect of the structure geometry on the absorption spectrum is analyzed. We suppose that only one parameter, e.g., the inner radius of the ring (r), the width of the vertical connecting bar $(\mathrm{w})$, the width of the horizontal bar (b), or the gap between the horizontal bar and the edge of the unit cell (s), is changed, while the other dimensions are kept the same. 
The simulation results for different $r, w, b$, and s values are presented in Figure 6. As shown in Figure $6 a$, with the increase in $r$, the resonance frequencies for both TE- and TM-polarized incident excitations are redshifted. An LC circuit mode for this metamaterial absorber is given by [26]

$$
\mathrm{f}=\frac{1}{2 \pi \sqrt{\mathrm{LC} / 2}} .
$$

The equivalent inductance $\mathrm{L}$ increases as $\mathrm{r}$ increases. It should be noticed that the increase in linewidth of metallic line leads to an increase in both the equivalent inductance $\mathrm{L}$ and the equivalent capacitance $\mathrm{C}$. However, the increase in equivalent inductance $\mathrm{L}$ is much stronger that the equivalent capacitance $C$ in this structure, as we mainly discussed the influence of equivalent inductance $L$ in this section. According to Equation (3), the increased L leads to a redshift of the resonance frequency. It can be seen in Figure 6a that the resonance frequency of TE-polarization, compared to that of TM-polarization, is more sensitive to $r$, which is because the resonance of TE-polarization is mainly concentrated in the ring area (Figure 4a); as a result, the resonance frequency of TE-polarization is more dependent on $\mathrm{r}$.

In Figure $6 \mathrm{~b}$, it is shown that, for TE-polarization, the absorption peak shows a slight blueshift with the increase in $\mathrm{w}$. This is because, in this case, $\mathrm{w}$ has little influence on the equivalent inductance $\mathrm{L}$ of the ring section. However, for TM-polarization, amplitude and frequency of the absorption peak are sensitive to the width $\mathrm{w}$, which can be explained by the fact that equivalent inductance $\mathrm{L}$ of the vertical bar decreases with the increase in $\mathrm{w}$.

Similarly, the increase in $\mathrm{b}$ leads to a decrease in the equivalent inductance $\mathrm{L}$ of the horizontal bar. Hence, the resonance frequency of TM-polarization is blueshifted (Figure 6c). However, this does not affect the equivalent inductance $\mathrm{L}$ of the ring area, so the resonance frequency of TE-polarization remains the same.

The effect of the gap s on the absorption spectrum was also analyzed, and results are presented in Figure 6d. As shown in Figure 6d, the resonance frequency of TE-polarization is independent of the gap s. However, with the increase in s, the resonance frequency of TM-polarization shows a slight blueshift, which is caused by a decrease in equivalent inductance $\mathrm{L}$.
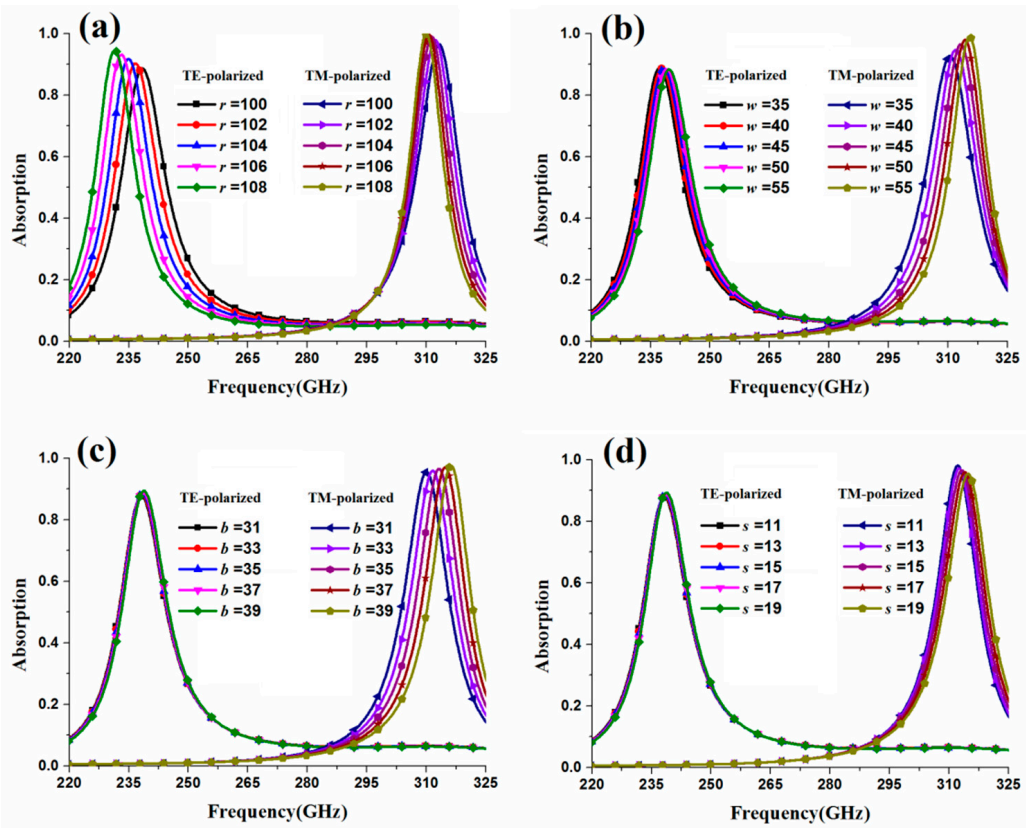

Figure 6. Simulated absorption spectrum dependence on different structural parameters: (a) the inner radius of ring, r, (b) the width of vertical connecting bar, $w,(c)$ the width of the horizontal bar, $b$, and (d) the gap between the horizontal bar and the edge of the unit cell, $\mathrm{s}$. 
In addition, the absorber structure was analyzed theoretically for oblique incidence. The absorption spectrum for oblique incidence under the TE-polarized incident wave is shown in Figure 7a. In this case, the magnetic field remains along the $y$-axis and the direction of wave propagation is changed by an angle $\varphi$ with respect to the $x$ and $z$-axes. Figure 7a shows a slight blueshift with the increase in incident angle and remains above 0.9 for an incident angle up to $60^{\circ}$. For oblique incidence under a TM-polarized incident wave, where the electric field is aligned towards the $y$-axis and the wave propagation is changed by an angle $\varphi$ with respect to $x$ and $z$-axes, as presented in Figure $7 \mathrm{~b}$, the absorption is almost unchanged for incident angles up to $30^{\circ}$. However, with the continuous increase in incident angle, the absorption decreases rapidly. Moreover, the increase in incident angle of the TM-polarized wave leads to an obvious redshift of the absorption peak, and a frequency tunability of $20.9 \%$ is achieved by increasing the incident angle from 0 to $60^{\circ}$, which represents another effective method of modulating the proposed structure.
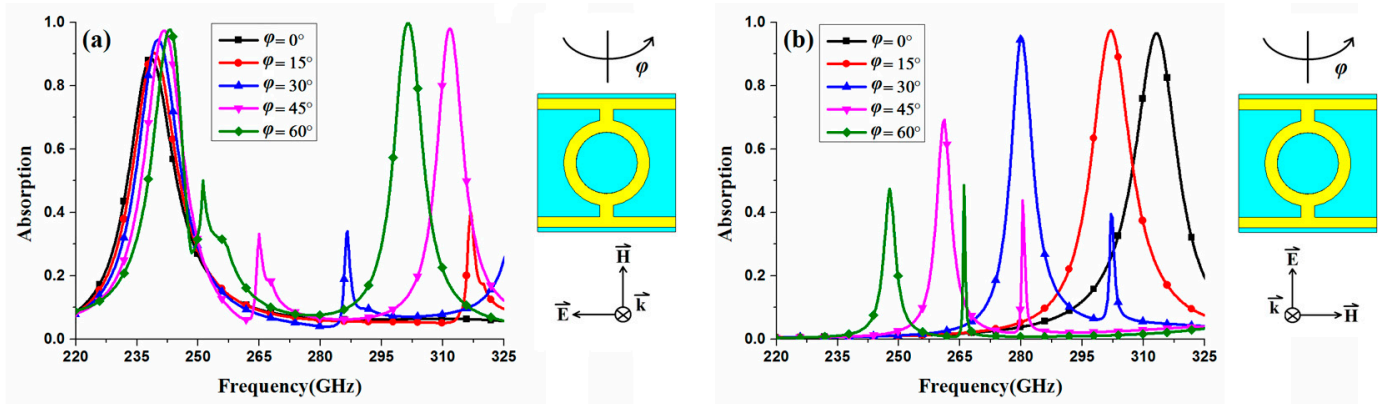

Figure 7. Simulated absorption spectra of absorber for different incident angles for (a) the TE-polarized incident wave and (b) the TM-polarized incident wave.

\section{Experimental Results}

In this section, we experimentally validate the proposed metamaterial absorber presented in Figure 1 . The structure was fabricated using $2 \times 2 \mathrm{~cm}^{2}$ quartz substrates. To control the orientation of LC molecules in an unbiased state, the inner surfaces of metal layers were covered by polyimide ( $\mathrm{Pi}$ ) alignment layers. The LC cavity was created by placing the glass micropearls with the diameter of $45 \mu \mathrm{m}$ between two wafers, which were then sealed by a thin film of epoxy resin. Lastly, liquid crystals were inserted into a $45 \mu \mathrm{m}$ thick cavity. The fabricated sample is shown in Figure 8.

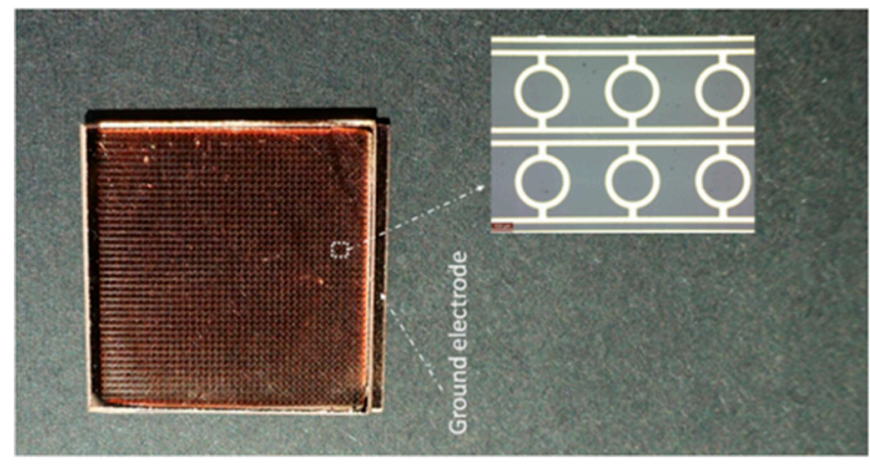

Figure 8. Structure of fabricated metamaterial absorber.

The measurement setup is presented in Figure 9. The free space measurement method was adopted in the experiment. An antenna that was connected to the Agilent N5224A vector network analyzer via VNA extenders VDI VNAX592 was used to measure S-parameters in the frequency range 220-325 GHz. Moreover, a pair of THz lens was utilized to focus the THz beam. 


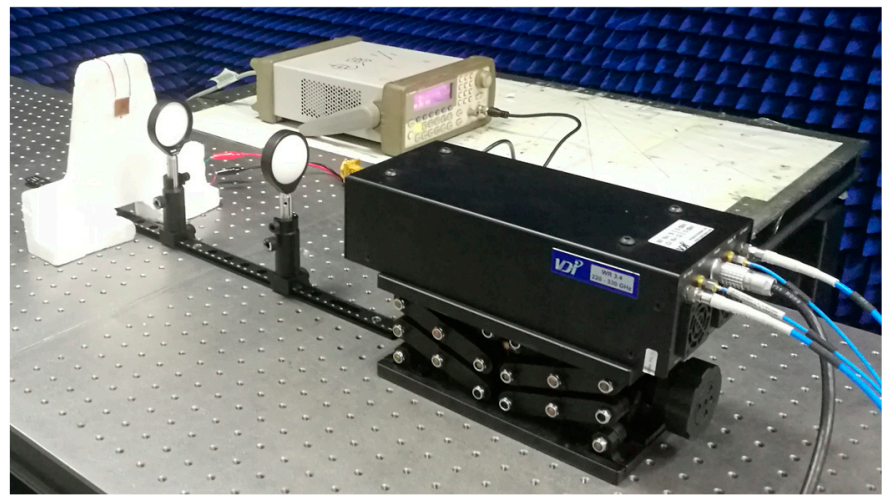

Figure 9. Measurement setup.

The measured absorption spectra at unbiased and full biased states for TE- and TM-polarization are given in Figure 10. For TE-polarization, Figure 10a, the frequency of the measured absorption peak in an unbiased state is in agreement with that in the simulation. However, in a biased state under TE-polarized incidence, the frequency of the measured absorption peak is higher than that in the simulation. For TM-polarization, Figure 10b, there is a difference between the frequencies of the measured and simulated absorption peaks in an unbiased state, which might be caused by an inaccurate sample size, especially for structural parameters such as the width of vertical connecting bar $w$ and the width of horizontal bar $b$.

(a)

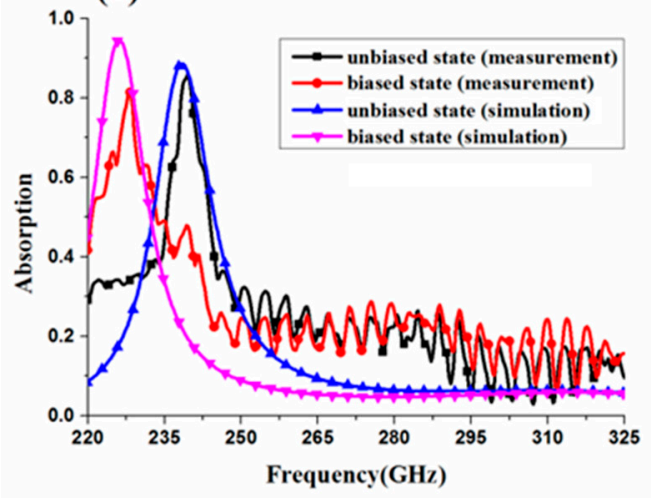

(b)

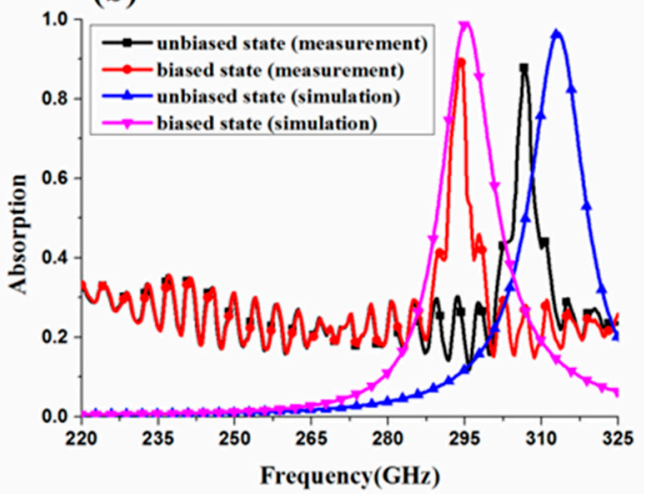

Figure 10. Simulated and measured absorption in unbiased and full biased states for (a) the TE-polarized incident wave and (b) the TM-polarized incident wave.

The frequency tunability was evaluated by measuring the absorption at different bias voltages of the LC layer. In Figure 11a, when the bias voltage increases from 0 to $10 \mathrm{~V}$, the resonance frequency of TE-polarization shifts from 239.5 to $228.1 \mathrm{GHz}$ (redshift), with a frequency tunability of $4.7 \%$. For TM-polarization, as shown in Figure $11 \mathrm{~b}$, a frequency tunability of $4.1 \%$ is achieved when the absorption peak frequency decreases from 306.6 to $294 \mathrm{GHz}$. Moreover, for both TE- and TM-polarization, measured absorption is higher than $80 \%$ for all bias voltages. 
(a)

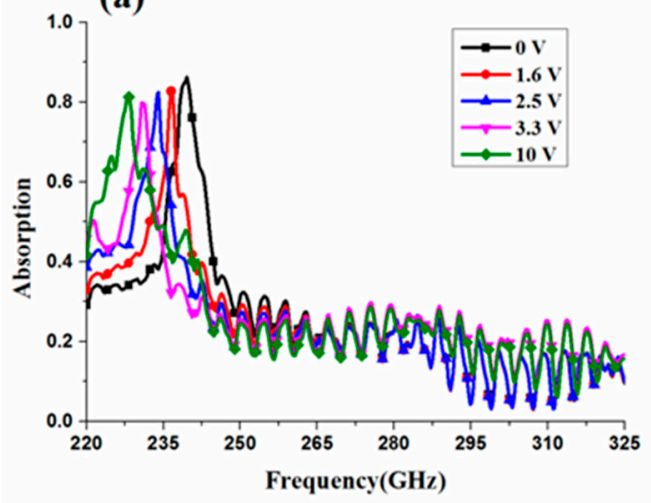

(b)

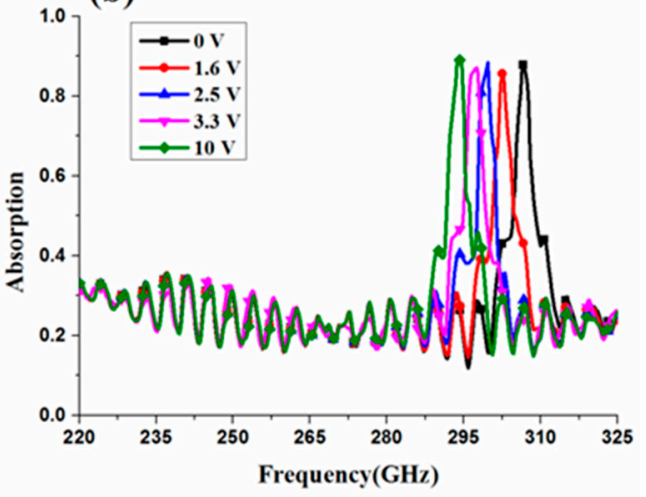

Figure 11. Measured absorption for different bias voltages for (a) the TE-polarized incident wave and (b) the TM-polarized incident wave.

\section{Conclusions}

In this paper, a tunable polarization-dependent metamaterial absorber based on LC intended for $\mathrm{THz}$ frequencies is presented, and both simulation and experiment were carried out to investigate the proposed structure. Intensive absorption peaks were experimentally detected at $239.5 \mathrm{GHz}$ for a TE-polarized wave and at $306.6 \mathrm{GHz}$ for a TM-polarized wave, when no bias voltage was applied. The absorption mechanism of the absorber was analyzed in terms of the surface current distribution and power loss distribution, and the effects of various geometrical parameters on absorption were investigated. Most importantly, by adjusting the bias voltage of the LC layer from 0 to $10 \mathrm{~V}$, a frequency tunability of $4.7 \%$ for the TE-polarized wave and frequency tunability of $4.1 \%$ for a TM-polarized wave were experimentally demonstrated. Therefore, the presented tunable polarization-dependent metamaterial absorber has great potential in selective spectral detection, polarization multiplexing, and $\mathrm{THz}$ polarization imaging.

Acknowledgments: This work was supported by the National Natural Science Foundation of China (No. 51607050), Fundamental Research Funds for the Central Universities (No. JD2017JGPY0006), and the Sichuan Science and Technology Support Project (No. 2016GZ0250).

Author Contributions: Guangsheng Deng and Jun Yang conceived and wrote the paper; Yujiao Lu, Zhiping Yin, and Hongbo Lu performed the experiments; Weien Lai guided the experimental design and analyzed the data; Aifeng Yang revised the whole manuscript; Yang Ye, Dayong Liu, and Baihong Chi gave important guidance on fabrication work.

Conflicts of Interest: The authors declare no conflict of interest.

\section{References}

1. Tonouchi, M. Cutting-edge terahertz technology. Nat. Photonics 2007, 1, 97-105. [CrossRef]

2. Hangyo, M. Development and future prospects of terahertz technology. Jpn. J. Appl. Phys. 2015, 54, 120101. [CrossRef]

3. Ferrari, L.; Wu, C.; Lepage, D.; Zhang, X.; Liu, Z. Hyperbolic metamaterials and their applications. Prog. Quantum Electron. 2015, 40, 1-40. [CrossRef]

4. Jung, J.Y.; Lee, J.; Choi, D.G.; Choi, J.H.; Jeong, J H.; Lee, E.S.; Neikirk, D.P. Wavelength-selective infrared metasurface absorber for multispectral thermal detection. IEEE Photonics J. 2015, 7, 1-10. [CrossRef]

5. Chen, Q.; Hu, X.; Wen, L.; Yu, Y.; Cumming, D.R. Nanophotonic image sensors. Small 2016, 12, $4922-4935$. [CrossRef] [PubMed]

6. Landy, N.I.; Sajuyigbe, S.; Mock, J.J.; Smith, D.R.; Padilla, W.J. Perfect metamaterial absorber. Phys. Rev. Lett. 2008, 100, 207402. [CrossRef] [PubMed]

7. Zhu, J.; Ma, Z.; Sun, W.; Ding, F.; He, Q.; Zhou, L.; Ma, Y. Ultra-broadband terahertz metamaterial absorber. Appl. Phys. Lett. 2014, 105, 021102. [CrossRef] 
8. La Spada, L.; Vegni, L. Metamaterial-based wideband electromagnetic wave absorber. Opt. Express 2016, 24, 5763-5772. [CrossRef] [PubMed]

9. Shen, Y.; Pei, Z.; Pang, Y.; Wang, J.; Zhang, A.; Qu, S. An extremely wideband and lightweight metamaterial absorber. J. Appl. Phys. 2015, 117, 224503. [CrossRef] [PubMed]

10. Yahiaoui, R.; Guillet, J.P.; de Miollis, F.; Mounaix, P. Ultra-flexible multiband terahertz metamaterial absorber for conformal geometry applications. Opt. Lett. 2013, 38, 4988-4990. [CrossRef] [PubMed]

11. Wang, B.X.; Zhai, X.; Wang, G.Z.; Huang, W.Q.; Wang, L.L. Design of a four-band and polarization-insensitive terahertz metamaterial absorber. IEEE Photonics J. 2015, 7, 1-8. [CrossRef]

12. Liu, S.; Zhuge, J.; Ma, S.; Chen, H.; Bao, D.; He, Q.; Zhou, L.; Cui, T.J. A bi-layered quad-band metamaterial absorber at terahertz frequencies. J. Appl. Phys. 2015, 118, 245304. [CrossRef]

13. Chu, J. K.; Wang, Z.W.; Guan, L.; Liu, Z.; Wang, Y.L.; Zhang, R. Integrated polarization dependent photodetector and its application for polarization navigation. IEEE Photonics Technol. Lett. 2014, 26, 469-472.

14. Sakurai, A.; Zhao, B.; Zhang, Z.M. Effect of polarization on dual-band infrared metamaterial emitters or absorbers. J. Quant. Spectrosc. Radiat. Transfer 2015, 158, 111-118. [CrossRef]

15. Wang, B.X.; Wang, L.L.; Wang, G.Z.; Huang, W.Q.; Zhai, X. Broadband, polarization-insensitive and wide-angle terahertz metamaterial absorber. Phys. Scr. 2014, 89, 115501. [CrossRef]

16. Kim, Y.J.; Kim, J.M.; Yoo, Y.J.; Tuong, P.V.; Zheng, H.Y.; Rhee, J.Y.; Lee, Y.P. Dual-absorption metamaterial controlled by electromagnetic polarization. JOSA B 2014, 31, 2744-2747. [CrossRef]

17. Ho, C.P.; Pitchappa, P.; Lin, Y.S.; Huang, C.Y.; Kropelnicki, P.; Lee, C. Electrothermally actuated microelectromechanical systems based omega-ring terahertz metamaterial with polarization dependent characteristics. Appl. Phys. Lett. 2014, 104, 161104. [CrossRef]

18. Deng, G.S.; Chen, P.; Yang, J.; Yin, Z.P.; Qiu, L.Z. Graphene-based tunable polarization sensitive terahertz metamaterial absorber. Opt. Commun. 2016, 380, 101-107. [CrossRef]

19. Shrekenhamer, D.; Chen, W.C.; Padilla, W.J. Liquid crystal tunable metamaterial perfect absorber. Phys. Opt. 2012, 110, 177403.

20. Isić, G.; Vasić, B.; Zografopoulos, D.C.; Beccherelli, R.; Gajić, R. Electrically tunable critically coupled terahertz metamaterial absorber based on nematic liquid crystals. Phys. Rev. Appl. 2015, 3, 064007. [CrossRef]

21. Lu, H.B.; Jing, S.C.; Xia, T.Y.; Yang, J.; Yin, Z.P.; Deng, G.S. Measurement of LC dielectric constant at lower terahertz region based on metamaterial absorber. IEICE Electron. Express 2017, 14, 20170469. [CrossRef]

22. Zhang, F.L.; Zhao, Q.; Gaillot, D.P.; Zhao, X.P.; Lippens, D. Numerical investigation of metamaterials infiltrated by liquid crystal. JOSA B 2008, 25, 1920-1925. [CrossRef]

23. Sasaki, T.; Noda, K.; Kawatsuki, N.; Ono, H. Universal polarization terahertz phase controllers using randomly aligned liquid crystal cells with graphene electrodes. Opt. Lett. 2015, 40, 1544-1547. [CrossRef] [PubMed]

24. Chikhi, N.; Lisitskiy, M.; Papari, G.; Tkachenko, V.; Andreone, A. A hybrid tunable THz metadevice using a high birefringence liquid crystal. Sci. Rep. 2016, 6, 34536. [CrossRef] [PubMed]

25. Khoo, I.C. Liquid Crystals: Physical Properties and Nonlinear Optical Phenomena; John Wiley \& Sons: New York, NY, USA, 2007; pp. 36-63.

26. Ye, Y.Q.; Jin, Y.; He, S. Omnidirectional, polarization-insensitive and broadband thin absorber in the terahertz regime. JOSA B 2010, 27, 498-504. [CrossRef]

(C) 2018 by the authors. Licensee MDPI, Basel, Switzerland. This article is an open access article distributed under the terms and conditions of the Creative Commons Attribution (CC BY) license (http:/ / creativecommons.org/licenses/by/4.0/). 